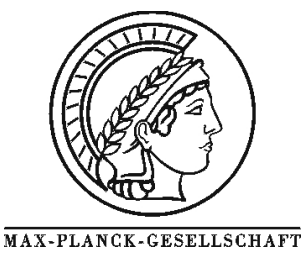

Crystal Research and Technology 2003, 38: 153-159

\title{
Crystallographic Shear Defect in Molybdenum Oxides: Structure and TEM of Molybdenum Sub-oxides $\mathrm{Mo}_{18} \mathrm{O}_{52}$ and $\mathrm{Mo}_{8} \mathrm{O}_{23}$
}

\author{
D. Wang ${ }^{*}$, D.S. Su, R. Schlögl
}

Department of Inorganic Chemistry, Fritz-Haber-Institute of the MPG, Faradayweg 4-6, 14195 Berlin, Germany

* Corresponding author: e-mail wangdi@fhi-berlin.mpg.de, phone +493084134482

Submitted 06 January 2003; accepted 14 January 2003

\begin{abstract}
Molybdenum trioxide and molybdenum sub-oxides are of great interests in catalysis due to their utilities as model system to elucidate the correlations between the structure and the catalytic performance. The sub-oxides are usually an intermediate phase during catalytic reaction in which the lattice oxygen is involved. We show the identification of the two common molybdenum sub-oxides $\mathrm{Mo}_{18} \mathrm{O}_{52}$ and $\mathrm{Mo}_{8} \mathrm{O}_{23}$, derived from $\mathrm{MoO}_{3}$ by crystallographic shearing (CS), by means of electron diffraction and High-Resolution Transmission Electron Microscopy (HRTEM) in combination with image simulation. The coincidence of simulated electron diffraction patterns and high-resolution images with the experimental ones indicates the feasibility of CS structure determination by these techniques.
\end{abstract}

Keywords: molybdenum oxides, crystallographic shear, electron diffraction pattern, high-resolution electron microscopy, image simulation.

\section{Introduction}

Molybdenum oxide based catalysts are extensively employed for partial oxidation of alkenes both in industrial application and in academic research. The reaction may proceed with interaction between species of chemisorbed hydrocarbon and oxygen by the so called Mars-van Krevelen mechanism, according to which the oxidation proceeds in two stages: (a) reduction of the catalyst with a hydrocarbon at the expense of the catalyst oxygen, which is incorporated into the products, and (b) re-oxidation of the reduced catalyst by gaseous oxygen. Recently it has been reported that not only the surface, but also the bulk structure of the catalysts is involved in the oxygen insertion process [1, 2]. For molybdenum oxides, consisting of $\mathrm{MoO}_{6}$ octahedra as their basic structural unit, the removal of oxygen species introduces considerable structural rearrangement. One of the ways in which oxides can accommodate such changes includes the well documented CS defect process. The CS essentially eliminates planes of anion vacancies by shear from corner to edge sharing of octahedra and the crystal collapse of the oxide, and therefore generates extended CS plane defects.

Superlattice of oxygen vacancies in $\mathrm{MoO}_{3}$ has been observed during thermal treatment in the electron microscope at higher temperatures and the oxygen vacancies were proposed to be consumed in the production of CS planes [3]. Furthermore, at early stages of $\mathrm{MoO}_{3}$ reduction using $\mathrm{H}_{2}$, $\mathrm{CO}$ or propene at temperatures varying from $\mathrm{RT}$ to $600^{\circ} \mathrm{C}$, partial screw dislocation in $\{101\}$ planes bounding a stacking fault extending from the surface, partial edge dislocation with Burgers vector $\mathbf{b}=[\mathrm{a} / 2, \mathrm{~b} / 7,0]$ forms on the surface. These reduction-induced defects can cause the lattice collapse and are consistent with crystallographic shear [4-6]. One consequence of the defect formation can be the formation of a short range ordered structure which was suggested to be " $\mathrm{Mo}_{18} \mathrm{O}_{52}$ " type crystallographic shear, as it was found by the recent in situ XAFS during TPR of $\mathrm{MoO}_{3}$ in $10 \mathrm{vol} \%$ propene from $300 \mathrm{~K}$ to $773 \mathrm{~K}$. The similar results were also obtained during the oxidation of $\mathrm{MoO}_{2}$ in oxygen [7]. Electron paramagnetic resonance study of interactions between 
$\mathrm{MoO}_{3}$ and propene confirms the formation of shear structures following desorption of the oxidized organic molecule $[8]$.

It is therefore clear that defects and defect formation play an important role in the red-ox process of molybdenum oxides, but the question, whether the defects are the driving force for the catalytic reaction or its by-products, is still open. In searching for the answers to the question, we have to first of all study the structure and formation of sub-oxides that are believed to act as an intermediate phase in cluster form during the red-ox process of the catalysts. In the present work, two representative molybdenum sub-oxides with CS structure, $\mathrm{Mo}_{18} \mathrm{O}_{52}$ and $\mathrm{Mo}_{8} \mathrm{O}_{23}$ are studied crystallographically in aspect of the formation. By matching the experimental high-resolution images and diffraction patterns with simulated ones, the CS structure determination can be achieved unambiguously. This also shows that the HRTEM technique combined with simulation can be applied to structure determination for other molybdenum oxides with different degrees of reduction.

\section{Structural principle of molybdenum oxides}

As well known, $\mathrm{MoO}_{3}$ crystallizes in orthorhombic structure (space group Pbnm). It consists of double layers of linked distorted $\mathrm{MoO}_{6}$ octahedra parallel to (010) plane. In each double layer $\mathrm{MoO}_{6}$ octahedra form edge sharing zigzag rows along [001] direction and corner sharing rows along [100] direction. Successive layers are held together by weak van der Waals forces only, with easy cleavage along (010) plane (Fig. 1). $\mathrm{Mo}_{18} \mathrm{O}_{52}$ can be derived from the layered $\mathrm{MoO}_{3}$ structure. It is convenient to project $\mathrm{MoO}_{3}$ onto

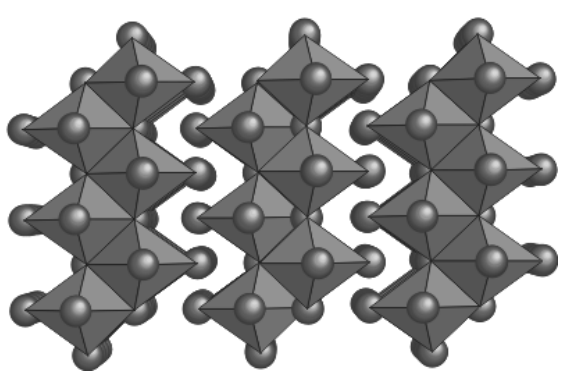

Fig. 1. [100] projected $\mathrm{MoO}_{3}$ structure model.

$(13 \overline{1})_{\mathrm{M}}$ plane, approximately the close packed plane. The removal of the rows of oxygen atoms from $(35 \overline{1})_{\mathrm{M}}$ plane is accommodated by the crystallographic shear operation along $\left[1 / 2 \mathbf{a}_{\mathrm{M}^{-}}-1 / 6 \mathbf{b}_{\mathrm{M}}\right]$, where subscript $\mathrm{M}$ denotes the vectors and planes of the $\mathrm{MoO}_{3}$ crystal [9]. This process is shown in Fig. 2a, where oxygen atoms stand on the point of each triangle and at the center of each dark triangle, one Mo atom is stacked on three oxygen atoms. The removed oxygen atoms are indicated by the circles. The formed $\mathrm{Mo}_{18} \mathrm{O}_{52}$ exhibits a triclinic cell and its [100] zone corresponds to the [ $\overline{1} 12$ ] zone of $\mathrm{MoO}_{3}$. Its [100] projected structure model is shown

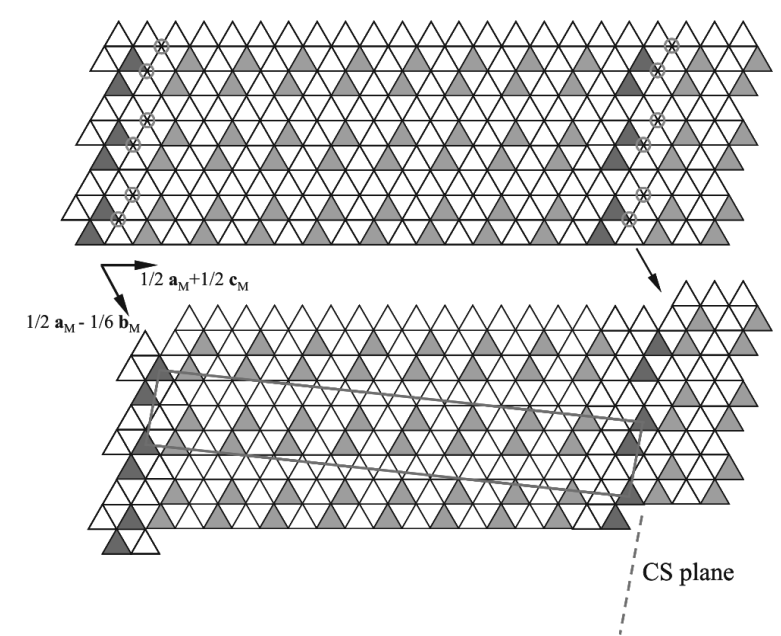

(a)

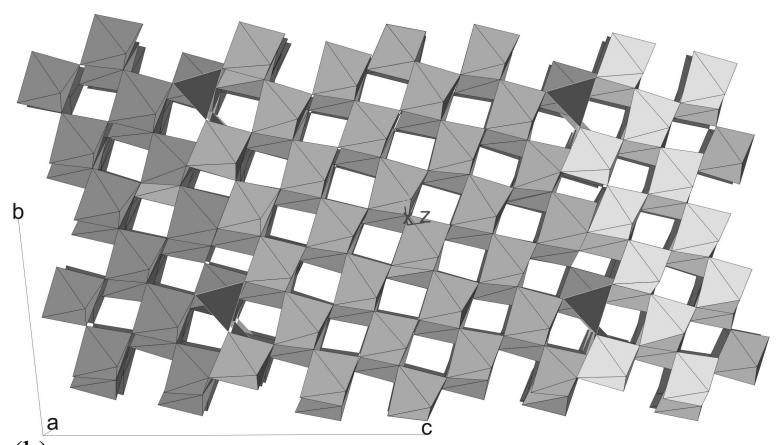

(b)

Fig. 2. Structural principle of $\mathrm{Mo}_{18} \mathrm{O}_{52}$. (a) CS planes formation exhibited on (13 $\overline{1})$ plane of $\mathrm{MoO}_{3}$ and (b) [100] projected $\mathrm{Mo}_{18} \mathrm{O}_{52}$ structure model.

in Fig. 2b. The space group of $\mathrm{Mo}_{18} \mathrm{O}_{52}$ is $\mathrm{P} \overline{1}$ with $a=$ $8.145 \AA, b=11.89 \AA, c=21.23 \AA, \alpha=102.7^{\circ}, \beta=67.8^{\circ}$ and $\gamma=110.0^{\circ}$

The $\mathrm{Mo}_{8} \mathrm{O}_{23}$ is formed by periodic crystallographic shear planes induced along $\{102\}$ plane of a $\mathrm{ReO}_{3}$ type matrix (Fig. 3). Thus formed CS planes are characterized by the

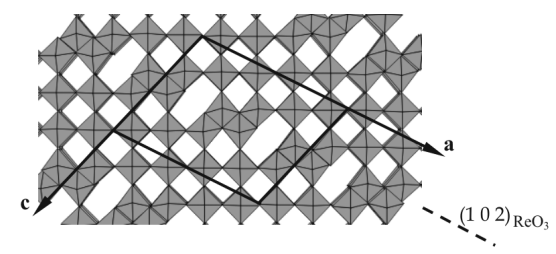

Fig. 3. [010] projected structure model of $\mathrm{Mo}_{8} \mathrm{O}_{23}$.

recurrent occurrence of groups of four edge-sharing $\mathrm{MoO}_{6}$ octahedra. $\mathrm{ReO}_{3}$ type blocks are between them, which extend through eight $\mathrm{MoO}_{6}$ octahedra. The [010] projected unit cell of $\mathrm{Mo}_{8} \mathrm{O}_{23}$ is also outlined in Fig. 3. The space group of $\mathrm{Mo}_{8} \mathrm{O}_{23}$ is $\mathrm{P} 2 / \mathrm{a}$ with $a=13.4 \AA, b=4.04 \AA, c=13.4 \AA$ and $\beta=106.5^{\circ}$. 


\section{Experimental}

The electron diffraction patterns (EDPs) and HRTEM images of single crystal $\mathrm{Mo}_{18} \mathrm{O}_{52}$ and $\mathrm{Mo}_{8} \mathrm{O}_{23}$ were taken on a Philips CM200 FEG microscope with accelerating voltage $200 \mathrm{kV}$, spherical aberration coefficient $1.35 \mathrm{~mm}$ and focus spread due to chromatic aberration about $3.0 \mathrm{~nm}$. The models of $\mathrm{Mo}_{18} \mathrm{O}_{52}$ and $\mathrm{Mo}_{8} \mathrm{O}_{23}$ were built using routine of "crystal builder" in Cerius package and the atom positions for these two crystals were derived from references $[10,11]$. The simulation of EDPs and HRTEM images was carried out using routines of "Diffraction-Crystal" and "HRTEM", respectively. Multislice method was used for electron propagation in crystal and each subslice did not exceed $2 \AA$.

\section{Results and discussions}

Fig. 4a shows the [100] projected electron diffraction pattern of $\mathrm{Mo}_{18} \mathrm{O}_{52}$. The simulated [100] electron diffraction pattern with crystal thickness of $40 \AA$ is shown in Fig. $4 b$. Both positions and relative intensities of the diffraction spots are similar to each other for experimental and simulated EDPs. The strong diffraction spots arise from the matrix and they coincide with the (110) and (02 $\overline{1})$ diffractions on [ $\overline{1} 12$ ] zone of $\mathrm{MoO}_{3}$ (Fig. 4c). Superimposed on the diffractions from the $\mathrm{MoO}_{3}$ matrix, other weaker diffractions can be attributed to the periodically distributed CS planes parallel to the (001) planes of $\mathrm{Mo}_{18} \mathrm{O}_{52}$, which correspond to the $\mathrm{MoO}_{3}(35 \overline{1})$ planes.

Based on the structure model of $\mathrm{Mo}_{18} \mathrm{O}_{52}$, [100] projected, simulated HRTEM images are calculated by $\mathrm{Ce}$ rius package with crystal thickness from $16.3 \AA$ to $195.5 \AA$ and with focus values from $-1400 \AA$ to $900 \AA$. Part of the simulated image contrasts are reproduced in Fig. 5. The focus step $100 \AA$ and the thickness step $16.3 \AA$ is suitable for exhibiting the distinguishable contrast difference. Figs. $4 \mathrm{~d}$ and $4 \mathrm{e}$ show two HRTEM images of obviously different contrast taken from different areas and with different defocus values. The insets in Figs. $6 \mathrm{a}$ and $6 \mathrm{~b}$ are the simulated images with thickness of $81.5 \AA$ and defocus $-400 \AA$, and that with thickness $32.6 \AA$ and defocus $-1000 \AA$, respectively. The contrast of the simulated images coincides with the experimental ones fairly well. The periodically distributed dark and light areas corresponding to the CS planes can be seen in Fig. 4d, while Fig. 4e shows rather even contrast. However, the diffraction patterns from these two areas are similar to that in Fig. 4a, which indicates the existence of CS planes. Therefore, to reveal CS planes directly in highresolution images depends on the conditions of image taking and image simulation needs to be carried out for structure determination. Since $\mathrm{Mo}_{18} \mathrm{O}_{52}$ keeps the layer structure and the cleavage usually takes place along bc plane, it is convenient to record the EDPs and HREM images on [100] projection and the CS structure can be determined with support of simulation.

Similarly, the [010] projected electron diffraction pattern of $\mathrm{Mo}_{8} \mathrm{O}_{23}$ in Fig. 6a shows the strong diffraction
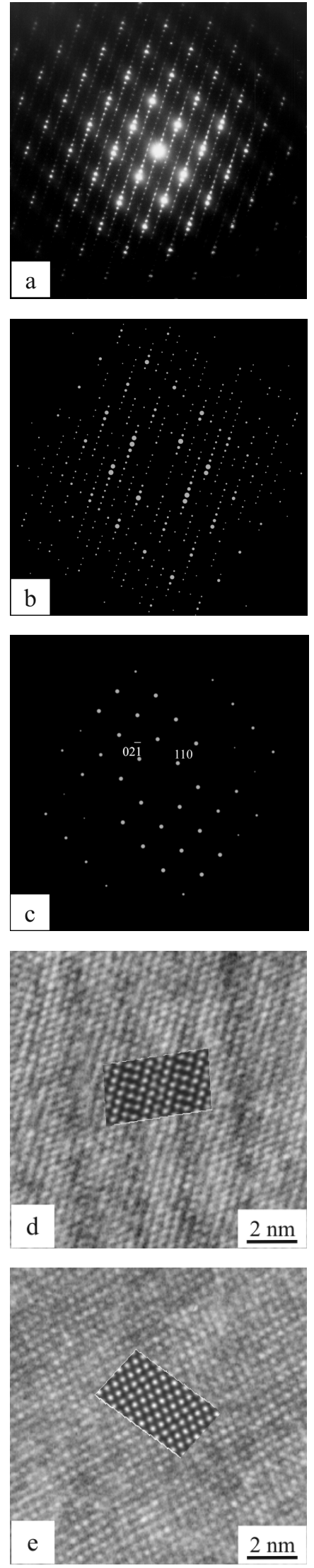

Fig. 4. (a) Experimental and (b) simulated electron diffraction pattern of $\mathrm{Mo}_{18} \mathrm{O}_{52}$ on [100] projection; (c) simulated electron diffraction pattern of $\mathrm{MoO}_{3}$ on [ $\overline{1} 12$ ] projection; (d) and (e) experimental HRTEM images of $\mathrm{Mo}_{18} \mathrm{O}_{52}$ on [100] projection with the simulated images insets. 


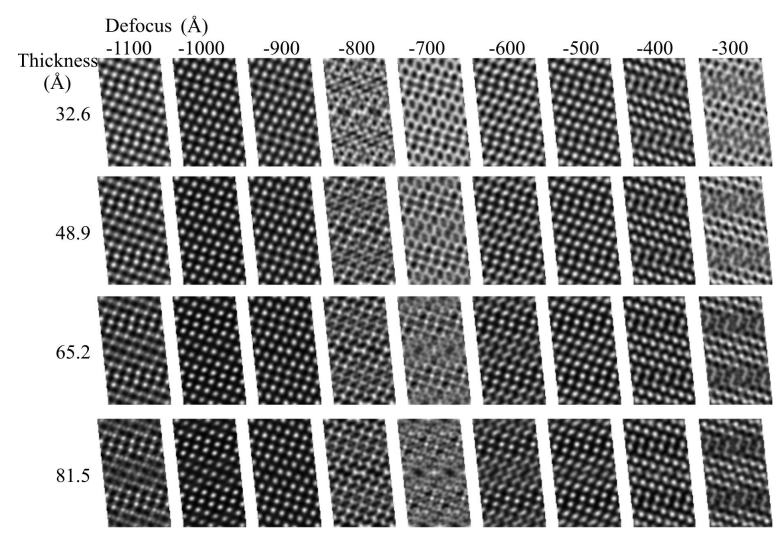

Fig. 5. Simulated HRTEM images of $\mathrm{Mo}_{18} \mathrm{O}_{52}$ with different crystal thickness and defocus values.

spots arising from the matrix with $\mathrm{ReO}_{3}$ structure and the satellite spots rows from the CS planes. Fig. $6 \mathrm{~b}$ shows the simulated electron diffraction pattern with crystal thickness $60.6 \AA$, which is identical to the experimental one. The corresponding high-resolution image is shown in Fig. 6c and the contrast directly indicates the existence of CS planes. [010] projected simulated images are calculated by Cerius package with crystal thickness from $40.4 \AA$ to $121.2 \AA$ (thickness step $20.2 \AA$ ) and with focus values from $-1400 \AA$ to $900 \AA$ (focus step $100 \AA$ ). Part of the simulated image contrasts are reproduced in Fig. 7. Among them, the image simulated with crystal thickness $60.6 \AA$ and defocus $-100 \AA$ (inset in Fig. 6c) matches the experimental image best. Another electron diffraction pattern is shown in Fig. 6d. The pattern can be regarded as a mirror reflection of that in Fig. $6 \mathrm{a}$ and the projection is deduced to be $[0 \overline{1} 0]$ direction. Corresponding high-resolution image together with the inset of simulated image with thickness $60.6 \AA$ and defocus -700 $\AA$ is shown in Fig. 6e. For $\mathrm{Mo}_{8} \mathrm{O}_{23},[010]$ or [ $0 \overline{1} 0$ ] projected EDPs and HREM images are suitable for CS structure determination since the characteristic can be easily distinguished in either of them.

\section{Summary}

$\mathrm{Mo}_{18} \mathrm{O}_{52}$ and $\mathrm{Mo}_{8} \mathrm{O}_{23}$ can be derived from $\mathrm{MoO}_{3}$ structure by different CS mechanisms. Our studies show that CS plane, which is important in understanding the oxygen diffusion and phase transition mechanism of transition metal oxides during catalytic reactions, produces well defined satellite spots in the electron diffraction pattern. This facilitates the application of TEM techniques for the investigation of the reaction mechanism in solid state chemistry. For $\mathrm{Mo}_{18} \mathrm{O}_{52}$, the CS plane in (001) plane, which is equivalent to $(35 \overline{1})$ plane of $\mathrm{MoO}_{3}$, produces regular spots on [100] projection, and for $\mathrm{Mo}_{8} \mathrm{O}_{23}$, the CS plane in (001) plane, which is equivalent to (102) plane of a $\mathrm{ReO}_{3}$ structure, produces regular spots on [010] projection, in the corresponding diffraction patterns. High-resolution imaging, taken along the proper crystallographic direction and supported by image simulation, allows the visualization of the CS structures at nano scale. This opens the possibility for in-situ HRTEM investigations of the reaction mechanisms under real catalytic conditions at atomic scale.
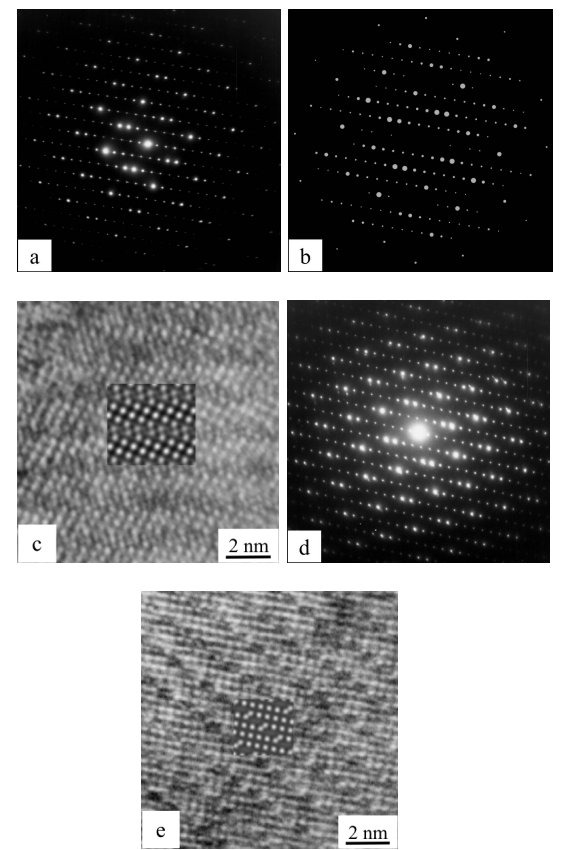

Fig. 6. (a) Experimental and (b) simulated electron diffraction pattern of $\mathrm{Mo}_{8} \mathrm{O}_{23}$ on [010] projection; (c) experimental HRTEM image of $\mathrm{Mo}_{8} \mathrm{O}_{23}$ corresponding to (a) with the simulated image inset; (d) experimental electron diffraction pattern of $\mathrm{Mo}_{8} \mathrm{O}_{23}$ in $[0 \overline{1} 0$ ] projection; (e) experimental HRTEM image corresponding to (d) with the simulated image inset.

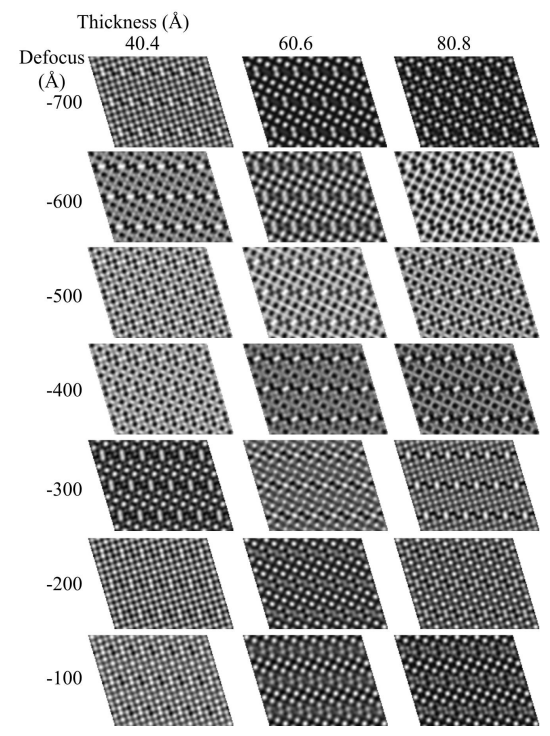

Fig. 7. Simulated HRTEM images of $\mathrm{Mo}_{8} \mathrm{O}_{23}$ with different crystal thickness and defocus values. 


\section{Acknowledgement}

The authors thank Mr. J. Jäger for helping TEM experiment.

\section{References}

[1] J. Haber, E. Lalik, Catal. Today 33, 119 (1997).

[2] B. Grzybowska-Swierkosz, Top. Catal. 11/12, 23 (2000).

[3] L.A. Bursill, Proc. Roy. Soc. A. 311, 267 (1969).

[4] P.L. Gai, Phil. Mag. A 43, 841 (1981).

[5] W, Thöni, P.B. Hirsch, Phil. Mag. 33, 639 (1976).

[6] W. Thöni, P.L. Gai, P.B. Hirsch, J. Less-Common Met. 54, 263 (1977).

[7] T. Ressler, J. Wienold, R. Jentoft, T. Neisius, O. Timpe, F. Girgsdies, Proc. XXXV. Jahrestreffen Deutscher katalytiker, Weimar 290 (2002).

[8] M. Labanomska, Phys. Chem. Chem. Phys. 2, 3307 (2000).

[9] L.A. Bursill, Acta Cryst. A 28, 187 (1972).

[10] L. Kihlborg, Ark. Kemi. 21, 443 (1963).

[11] A. Magneli, Acta Chem. Scand. 2, 501 (1948). 